\title{
UNISEX CLOTHING: A SUSTAINABLE WAY OF SELF EXPRESSION
}

\section{CHETNA DIKKAR}

Assistant Professor, Department of Fashion Design, MGM University, Aurangabad, India

\begin{abstract}
In the recent times the fashion industry has moved its focus towards sustainable fashion with an aim to pace down the overall production on a global scale and thereby reducing the demand and consumption of fashion products. The movement of reduction in pace also known as 'Slow fashion' promises to trim down the enormous textile waste, restore environment, bring back ethical working conditions in fashion industry and make the industry sustain in the long run. Along with changing manufacturing and selling practices, there is still a need to change the individual clothing habits to make an outsized difference. The current COVID 19 situation has given us a glimpse of minimalism as way of life. People during lockdown experimented with what they had, swapped and shared clothes and refrained from buying new due to the uncertainty of financial future. With no specific point to prove, consumers chose unisex and gender fluid clothing options for the sole reason of comfort. This pandemic can be seen as an opportunity for reconditioning the mind-set towards traditional clothing and buying habits. Like never before the today's consumer is equipped with digital exposure and knowledge in order make right purchase decisions that would eventually change the course of the current fashion scenario. This change in paradigm is studied and documented based on the information from secondary sources.
\end{abstract}

KEYWORDS: Sustainable fashion, Minimalism, Consumer habits \& Unisex clothing

Received: Feb 19, 2021; Accepted: Mar 11, 2021; Published: Mar 19, 2021; Paper Id.: IJTFTAPR20212

\section{INTRODUCTION}

Even though the awareness of sustainability had been in existence for decades, 'Sustainable fashion' seems like a fairly new concept. In the early 90's visionaries for the first time tried to break free the stereotype of the compressed fashion industry by innovating ways and means other than the existing practices of manufacturing and consumption. Ozdamar (2015) elaborated on the fact that clothing can serve as tool to maintain ecological, social and cultural diversity.It further encouraged innovative business models that could transform into substantial movement to move away from the growing trend of fast fashion.

Rapid consumerism and globalisation has fuelled the drift of having the latest trends at a very short span of time in very affordable prices. This has been only possible due to mass production compromising on all ethical practices of production and consumption, thus posing a threat to the sustainable fashion movement. The main focus of the fashion production houses is to meet the enormous demand of affordable clothing at a very short span of time. While doing so many unscrupulous ways are employed which in turn lead to abuse of working conditions, exhaustion of natural resources, deteriorating the eco system and creating a massive textile waste dump. To deal with the glaring concerns of fast fashion, Sustainability has become the latest trend, all websites, establishments and brands are talking about and are taking initiatives to maintain transparency with their customers about their production practices and its impacts on environment. 
Today's consumer is more aware of the product, its manufacturing process and of its fate in the ecosystem. There is information easily available on alternatives to everything; as a result the sustainable fashion has gained momentum. However it is easy succumb to the old ways of readily accessible, affordable and mass produced clothing. Though sustainable or slow fashion seems to be ethically correct, it is not affordable to all consumer segments due to the time and practices involved in its production.

In order to reset the never ending cycle of use and discard, one needs to resort to the traditional sustainable practices of clothing. Until the last century 'hand-me-down' was an integral part of every household. Clothes had long life cycle as they were treated as commodities of value which are limited and not frequently purchased. Pre-used clothes were passed to one another from older siblings and thus normalising slow fashion. This sustainable mind-set if reinstated could embed sustainability on a fundamental level. Hands me down as a culture has also disappeared due to small families with fewer members and if we add the gender variant there is very minimal possibility of swapping and reusing clothes. However with evolution of ideologies of gender fluidity, people waking up to elimination of old binary structure of gender identity and are making individual buying decisions according to their own suitability and preference. One such concept that aids this revolution of fashion choices is 'Unisex clothing'.

Fashion has always been a way of self - expression and individualism throughout the ages. For ages the gender identity has been well-thought-out to be either male or female, with rise of the unisex fashion these gender barriers are now collapsing. Designers all over the globe are identifying with self-expression over age old norms. Today's consumers are well aware of their creed and see fashion as an opportunity to express their individuality. Gender and sex which have been considered as one initially now have layers of differentiation difficult to comprehend. In this chaos, unisex clothing serves as a medium for expression of all. Unisex garments are designed with silhouettes keep in mind no specific gender but the functionality of the clothing element.

With the current fast fashion crises styles and trends in the apparel market are short-lived; this keeps the industry in a constant demand mode for new. The need of the hour is to create and evolve styles which are easy to manufacture and have minimum categories to be on-shelf and promoted. Unisex garments fit this bill completely with reduced design detailing and no gender variants. On the other hand consumers too want style solutions that reduce the shopping time and enable a common wardrobe amongst people in the family. The reasons illustrated so far have led to the emergence of a new category in apparel industry i.e. Unisex clothing. Major fashion analysts, researchers, fashion and trend forecasters feel that Unisex clothing is going to be one path breaking ideologies which will be trending for a while. The dawn of which is already seen in form of major internationals designers launching their Unisex lines. India being multi-cultural diverse country the unisex clothing is emerging in simpler categories of sportswear, casuals, lounge wear and sleepwear.

\section{RESEARCH METHODOLOGY}

The research methodology of this paper is an exploratory study based on secondary data, obtained from secondary sources. Data is collected through various research papers, books, websites, published research thesis and blogs etc.

\section{DEFINITIONS}

Soones and Stevenson (2005) defined 'Uni' as a connotation for one or a combination to comprise a single form. 'Uni' as a prefix stands for single and 'sex' refers to biological differences between men and women. Hence 'uni' when combined with 'sex' stands for one sex. 
Based on these definitions Unisex Clothing can be understood as fashion that is designed and made to be worn by either of the sexes.

Callan (1998) refers unisex fashion as clothing designed in order to be suitable for both sexes, bring about appearance of men and women to be similar and rendering the idea of sexuality vague.

According to Kaiser (1997) Gender implies a social construction, a tool that is used to express peoples ${ }^{\text {ee }}$ perceptions of what they think it means to be male or female.

Kaiser (1997)refers 'sex' to the biological anatomy; differences between male and female.

However, 'sex' is synonymous with biological differences, whereas 'gender' has social and cultural connotations as there are different social norms from men and women.

Thus Unisex clothing is more of a gender independent design than 'single sex' or 'sex-less' design. It is designed on the common ground of silhouettes and design elements that work for both the sexes.

Fashion has always been a mirror of one's identity and individuality. According to Paoletti (2015) numerous aspects influence personal style such as age, class and ethnicity, but gender and sexuality has been the dominant through time.

\section{UNISEX CLOTHING}

Humans, the only known creatures to have created clothes, have also evolved and adapted the art of clothing ever since its inception. Clothes are articles used to cover and protect the body from the harsh conditions of the environment. Though the fundamental function of the clothing was protection and modesty, with time it morphed to others such as depicting social status and affluence. Barbara Vinken (2004) explains that fashion at a principal level is intended to show a conflict. It has been governed by the conceptual divisions i.e. being or mere appearance sexes and classes. For ages mankind has been categorised into two major sectors men and women based on their sex and controlled by the associations attached to each. As Freud expressed, whenever you meet someone, the first assumption you make is male or female. Vinken further elaborates that the divide by sexes in fashion was at its peak in $19^{\text {th }}$ century. Early 1960's was a period of social sexual renaissance in form of political movements. Ever since then major changes have happened in the fashion sector with blurring of line between men's and women's silhouettes. The elements of clothing are independent of gender, if a guy wears it, it becomes masculine and if a girl wears it she makes it feminine. Hence gender in clothing is nothing but a socio cultural perspective. Unisex clothing has neither male nor female attributes; they are silhouettes which can work for both genders based on individual interpretation.

In an American weekly magazine (1883-1972) the term unisex was first used. The unisex trend in west gained momentum in the 1960s as it became bid on the Paris runways where 'Space age' inspired collections by major designers like Pierre Cardin, PacoRabanne and Andre Courreges were showcased sleek silhouettes in synthetic fabrics with no gender implications. In 1969, Burning of Bras (a symbolic representation) by women in U.S. created a wave of liberation and emergence of unisex sections in departmental stores. These events through small had a ripple effect on the fashion trends even decades later. The 60s and 70's raised many questions about sex and gender but never fully answered any.

Jo Paoletti (2015) gives an elaborate analysis of fashion and sexual revolution. Paoletti writes that even though unisex clothing is intended to minimize gender familiarities it had reverse effect on its onset. The space age inspired unisex 
clothing of the mid 70s, though gender neutral were tight fitting made out of synthetic stretchable fabrics which made the gender of the wearer even more conspicuous. When the unisex trend resurfaced in the 1990s it was more mainstream with silhouettes appeals both sexes alike.Women were seen in flannel lumberjack shirts and war boots. Fashion icons like Kurt Cobain sported ball gowns. Around the same time, Keo-Peul-Look a fashions that gained attention as lookalike couples fashion in South Korea. In a country where open display of affection received cold shoulders, the new version on "his-nhers" fashion gained popularity. Koreans adorned easily accessible unisex garments like sweaters, skinny jeans, sneakers and hoodies. Space Age made a comeback with Dior's fall 2013 couture in the form of techno jumpsuits and Gucci showcased modern shift dresses with leather with an underlying idea of dissolution of genders.Even haute couture was made unisex for a Spring/ Summer 2015 collection by Rad Hourani.

In India, though unisex clothing had been a part of the traditional costume as draped lower garments, its acceptance in contemporary mainstream was limited. However this trend has been sported everywhere by many celebrities these days with no signs of disappearing soon. Major fashion brands like Allen Solly, Koutons and Aditya Birla have entered the unisex clothing segment with great merchandise. This serves as a great opportunity for men and women to experiment with new silhouettes and figure out their preferences. Although many established brands started segments for unisex clothing, it was not until the recent times that stand alone gender independent labels with a unique brand identity were launched. Currently there are brands catering solely to the unisex segment such as HUEMN, KallolDutta, Nor Black Nor White, Anaam, The Pot Plant, Bobo Calcutta and Bloni. With limited brands in this segment, the brands fall under affordable luxury which isn't within the normal consumer reach.

Clothing items that do not have a gender connotation attached to it easily fall under unisex clothing such as tshirts, blue jeans, vests, sweaters, shirts, oversized blazers, lounge pants etc. As opposed to gender specific clothing which has a silhouette emphasis to highlight a particular gender trait or have decorative details that are associated with a particular gender. Unisex clothing is designed on the principal of striking a mean between the details of masculine and feminine clothing elements to create silhouettes that work comfortably for both genders.

Fashion has an extensive, diverse and artistic history. All throughout time, fashion of a particular period has been nothing but reflection of the socioeconomic sentiments of that period of time. Trends often largely echo the sentiments and emotions of the society at a given time. Theorists feel that for ages there has been an intentional representation of ultrafemininity andmasculinity to aid modern marketing and prosperity of individual sectors. Unisex clothing serves an opposition to this normal course of age old fashion flow. The unisex fashion is a fashion forward movement allowing individuals to establish their fashion preferences.

\section{GENDER CONFLUENCES IN HISTORY}

According to Vinken fashion creates boundaries, but strangely it also wipes away the separation of sexes and then affectedly shows the hints of the division that was once there. Eicher and Evenson (2014) further elaborates that sometimes society highlights sexual characteristics and at other times de-emphasises them based on the social atmosphere of that period.

\subsection{Androgyny}

The term 'androgyny' is an amalgamation of Greek words for male (andras) and female (gune) and stands for somewhat amid masculinity and femininity. According to Soones and Stevenson (2005) androgynous appearance is a blend of the 
binary aesthetics, depicting mixed sex ideologies for male and female. Earliest signs of androgyny were seen in the early eighteenth century in England and France in the form of restricted androgynous fashion. During 1800s due to industrial revolution uniforms were produced on a large scale. Androgyny got apush when female spies were recruited in the military which lead to uniforms that comprised trouser and jacket to be worn over dresses. When women started entering varied job profiles, they preferred men's silhouettes for comfort and ease of movement.

Warren (1980) explains that androgyny represents a retreat from gender restrictions, from socially imposed prejudices of norms for women and men. Androgynous fashion had a rebellious edge which fascinated the women. Again in 1850s 'the Bloomers' that gained a lot of media attention introduced by Amelia Jenks Bloomers were a form of pantaloon pants. These were referred as men's pants made especially for women. Women all over the world started making enquires about this style and were more than eager to get rid of the weight of long skirts. Around World War lin the early 1900s designers like Coco Chanel and Paul Poiret introduced 'Flapper fashion'. This flapper style was endorsed by a lot of celebrities was a new take on androgyny with short bob hair and trousers for female. Worsley (2019) illustrates that 'Le Smoking' a sleek tailored suit by YSL in 1966 finally sealed the acceptability of suit as a substitute to skirt/ dress. Yohji Yamamoto and ReiKawakubo, Japanese designers in 1980s used fashion to challenge social assembles of gender ideas through their androgynous designs. By the early $21^{\text {st }}$ century androgynous fashion became globally accepted with lot celebrities endorsing it.

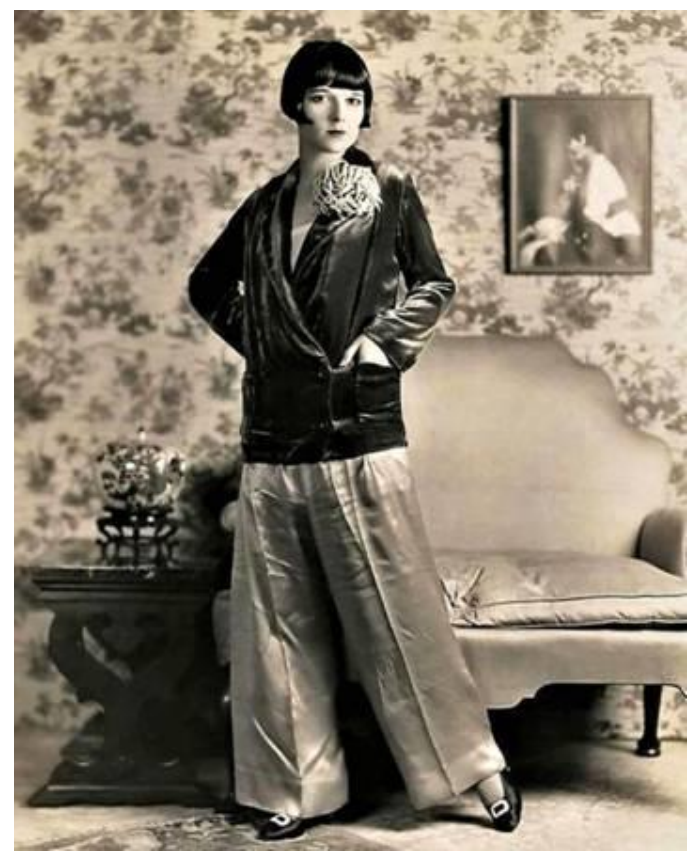

Figure 1: Androgyny Post WW-I

\subsection{Metrosexual Man}

There has been no substantial change in men's fashion for centuries regardless of their evolution. Men's fashion has changed at a very gradual pace with the underlying idea of functionality and comfort. But there are instances in history like Ancient Assyria, Greece, Rome and the Middle Ages, where men from the royal family and higher ranks groomed themselves and dressed lavishly. These are the periods where ornate elements, drapes, jewels and even make up was a part of men's fashion. In the seventeenth and eighteen century men's fashion evolved according their profession with just few changes around it. Minimalism in a man's clothing was a reflection of his conduct of work. The idea of aestheticvisibility 
was never a key motivation in men's fashion during this period. Later after a long gap the trend of fashion aware man came back in 1900s when Hollywood became big with larger than life male characters were portrayed on the silver screen.

Historically the King played a major inspiration for popular styles of clothing as it was immediately followed by his subjects. Likewise in the modern world Hollywood personalities, Sports icons, World leaders and Artists influence the fashion of the generation. Metrosexual man is a term used for a well groomed young man with a good amount of disposable income living in a city and well aware of his image. This term surfaced in 2002 by Mark Simpson in his article 'Meet the Metrosexual', where he describes the emergence of new breed of men who consciously look after themselves, are well dressed, and mindful of their physique. A trend in which image became more significant than identity. This kind of a man became every marketer's dream, men's beauty and grooming industry flourished like never before. Metrosexual mam was experimental and was more open to design aesthetics which would not please existing gender norms. Like tight head to toe leather dressing brought in vogue by the rock bands in the 1960s. This period in men's fashion was a complete metamorphosis of the minimal gender restricted male wardrobe and men's styles continue to evolve like never before. With more women on the work front and fading gender roles, men are paying attention to their choices in clothing; this has led to diversification of existing categories in men's fashion. Even men are now more concerned about their individuality and authenticity.

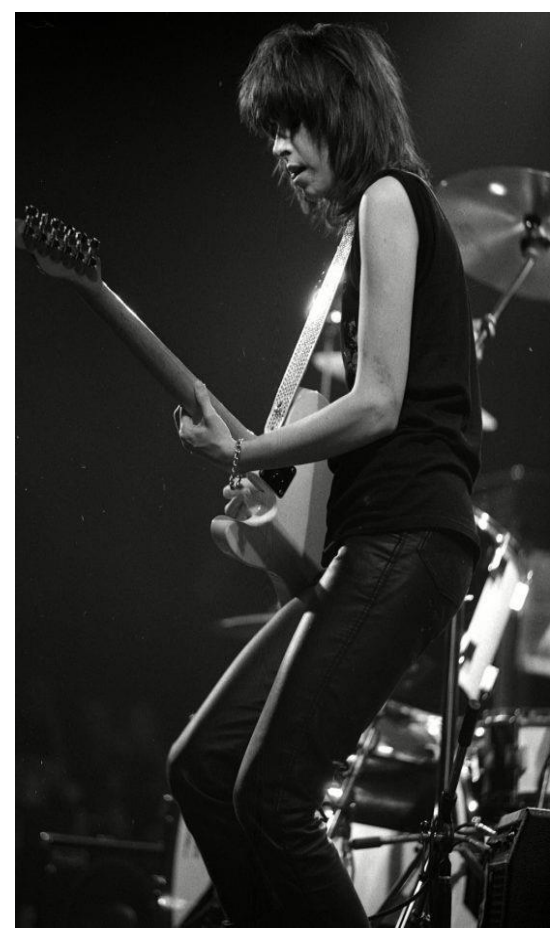

Figure 2: Fashion Influenced by Rock Band Culture 1960s

Today's world is a dawn to new identities leaving behind archaic gender structure and male- female behavioural norms. There is major blurring in predefined gender roles, man is taking interest in a lot of lifestyle activities and has become more self-aware in terms of grooming and image. Women on the other hand are no longer confined to household work, are counterparts of men at work and have financial independence to take their decisions. With the understanding of new gender divide and its crossovers to some extent, has brought about opportunities of new fashion solutions that are neither male nor female but represents the current surge of the society. 


\section{FACTORS INFLUENCING CURRENT CONSUMER BUYING CHOICES}

\subsection{Fast Fashion}

Today in the digital when products are seen, desired and bought at a pace of a click, consumers have grown into a challenging fashion demand mode. This high demand has to be fulfilled in a short time with an intent not losing the market to numerous competitors. This mode of instant gratification of fashion demands is termed as 'Fast Fashion'. Fast fashion brands are extremely flexible to consumer demands and capture current trends from international catwalks and deliver it to consumers at an amazing pace. Fast fashion works on the understanding of segmented consumer market; as a result numerous players have entered working on the principle of fast fashion that provides products of different niches.Traditional buyers which are a very limited portion of the current consumer segment buy limited fashion based on fashion calendar according to the seasons. However a major portion of fashion consumers still depend on on swift supply chain of fast fashion.

The introduction of fast fashion has broken the two season monopoly of fashion retailers and has led to emergence of many individual small scale fashion retailors. Fashion though not custom made has become more consumer centric. On one hand where the consumers have become peckish for different each time on the other they want more from whatever they buy. Consumers are looking for more end use options of a single product. This could also be seen in the rise of many multi-utility segments in clothing such as Athleisure, where the garment is supposed to function beyond its function as sports attire. This aids the acceptance of versatile unisex clothing.

\subsection{Concept of Self}

Viken (2004) elaborates that fashion serves the cause of distinction and at the same time that of equality, making it possible for individuals to stand out and yet at the same time reference their social belonging. Every individual has an innate understanding of what they like or dislike. Similarly they have preferences in terms of accepting the fashion choices offered to them. Eicher and Evenson (2014) explain that other than physical appearance, the factors that decide choice of an ensemble are personality, mood, abilities and preferences. The current age is flooded with digital influencers, every individual is idolising someone or the other with a view of finding their own personal style. The idea of accepting individualism is becoming significant like never before. Consumers no longer want to dress like others but want to use fashion as medium to express their individuality. They are trying to find styles that work best for them keeping in mind factors like preference, comfort and functionality. Some individuals believe that should not be obvious but instead subtle. Customisation is need of the hour based on individual tastes and inclinations. Consumers are willing to take risks to assert their uniqueness.

\subsection{Body Positivity}

Throughout complex history of art and fashion there has been an ideal size for everything. This aspired size has never been constant but a reflection of societal whims at particular period of time. Body image is how one perceives their physical self. Body image is a very individual notion as it is shaped out of individual reminiscences, experiences and theories. Body positivity is a movement that started in 1990s with an aim to break free from the societal body image standards. The term 'body positive' a term coined in 1996 to shift the focus of people trying to lose weight through unhealthy means. With numerous mental health issues being associated body image, the body positivity movement has again gained momentum. 
The neo body positivity advocates inclusiveness of people from all sizes, colour, race, gender and sexuality. This heightened sense of freedom from ideal body constraints has made way for silhouettes in clothing that work for all.

\subsection{Gender Neutrality}

In order to tackle the issue of gender inequality and discrimination, a social movement called gender neutrality has gained impetus. Gender neutrality works on the notion of creating policies, opportunities and obligations devoid of the sex or gender divide. It further advocates on diminishing old norms of gender roles foe men and women. With the society moving towards inclusiveness, the binary structure is slowly collapsing and making way to key roles being decided on ability and merit. Emphasis has been laid on cultivating gender neutrality as a concept very early on in education, so the perspective gets imbibed in the young minds to an degree where male and female are just an expression and signify the same.

\subsection{Product Longetivity}

In order to minimise the environmental consequences of product dumping and extending product life cycle, product longevity play a key role. The manufacturing, usage and disposal are the main phases of a product life cycle. By extending the usage phase of the product not only delays its end disposal but also curbs the demand of its substitute. Extending longevity can be achieved through design changes, repairs and re-purpose of the product. In clothing re- purpose is not a novel concept and has been a part of many age old traditions. Product design practices and marketing strategies can educate consumers to bring re-purpose in vogue. Product should be designed with a view to extend usage phase of the product cycle and its dynamic functionality.

\subsection{Consious Consumerism}

2010s is when conscious consumerism came into picture; due to the wide spread awareness of environmental hazards caused by the clothing and textile industry. Numerous measures have been taken since then to ensure sustainable fashion such as transparency in production practices, consumption with minimal ecological damage, zero waste, ethical labour practices, free from animal cruelty, material innovation and recycling. Minimalist fashion is one such individualistic approach towards conscious consumerism. The idea of minimalist wardrobe is to invest in high quality versatile pieces of clothing that can be re-purposed and worn as many times as possible. Sustainable fashion should be such that it caters maximum consumers with minimum choices. Unisex clothing works on the principle of minimalism with gender fluid aesthetics and multi-function dynamics.

\subsection{Post Covid Lifestyle}

Like any other industry fashion has been majorly affected by the COVID pandemic and has undergone many changes. A new form of fashion has evolved to catch up with the changing stay at home concept. Categories such as atheleisure, breezy silhouettes, lounge wear and sleep wear have become popular. Work from home has reduced the pressure of office dressing norms and scope for experimentation. Economic crunch has slowed down purchases and has brought repurposing of existing clothing to the forefront. Fashion elements are styled and reused to create new looks from limited wardrobe pieces. Pandemic has brought back the importance of wardrobe stables i.e. is key pieces of quality clothing. With everyone at home wardrobe have been swapped and experimented like never before. During the pandemic detached from the world outside fashion became a private affair and opened doors of self expression. Comfort took over design aesthetics and individuals learnt the art of dressing for themselves. 


\section{CONCLUSIONS}

Sustainable fashion is growing segment of the clothing business and could flourish even more in future with increasing awareness of environmental hazards caused by production practices and unethical working conditions. In order to make the fashion industry more sustainable for future the consumption and production practices of fashion products need to be rethought upon. Though this has to happen in the bigger picture, on an individual level minor changes such as slowing down consumption can aid sustainability in the long run. Product design should now look beyond the basic parameters of form and function to create products that are multi-purpose and have longevity. Consumers should be made to think beyond 'green' and 'eco-friendly'. This awareness and options for change should be communicated to the consumer level by developing innovative marketing approaches. The current trend of functional clothing over short lived fast fashion should be encouraged and continued, as the segment of functional clothes surpasses all limitations of conventional clothing.

The concept of unisex fashion is getting accepted in Indian markets and designers are creating collection around it. Fashion forward consumers are not questioning its flight from normal and are willing to experiment it as a fashion statement. The abrupt changes in the world in light of the current COVID crises have made it obvious that the design focus has to shift its focus from commercialism to survival. Unisex clothing can be a new approach to sustainable fashion based on the principles of effortless silhouettes working for all, hands me down tradition, wardrobe sharing and minimalism. It can serve as a tool of self expression by breaking free from the binary norms and exploring individual choices based on comfort and functionality.

\section{REFERENCES}

1. Barbara Vinken. (2004). Fashion Zeitgeist: Trends and Cycles in the Fashion System. Berg Publishers - Bloomsbury.

2. Harriet Worsley. (2019). 100 Ideas That Changed Fashion. Laurence King.

3. Joanne B. Eicher \& Sandra Lee Evenson. (2014).The Visible Self: Global Perspectives on Dress, Culture and Society. Fairchild books. Bloomsbury.

4. Jo B. Paoletti. (2015).Sex and Unisex: Fashion, Feminism, and the Sexual Revolution. Indiana University Press.

5. Gopalkrishnan Shrinivasan. (2017). Awareness on Unisex Clothing among Fashion Designers: A Study. International Journal in Management and Social Science. Vol.05. Issue-02.

6. Najmul Kadir Kaikobad. 'et al'. (2015) Sustainable and Ethical Fashion: The Environmental and Morality Issues. IOSR Journal of Humanities and Social Science. Vol 20. Issue 8.

7. Annamma Joy \& Camilo Pena. (2017).Sustainability and the Fashion Industry: Conceptualising Nature and Traceability. The University of British Columbia. Academia.edu.

8. K. Archana Ranjan. Unisex Clothing Trends \& Rationale. National Institute of Fashion Technology: Rae bareli. Academis.edu.

9. Kimberly Chrisman-Campbell. (2015). A Brief History of Unisex Fashion. The Atlantic. Retrieved from https://www.theatlantic.com/entertainment/archive/2015/04/when-unisex-was-the-new-black/390168/

10. Aidan Russell. (2020). The History Of Androgynous Fashion Up To Contemporary Times.Fibre2fashion.com. Retrieved from https://www.fibre2fashion.com/industry-article/8750/the-history-of-androgynous-fashion-up-to-contemporary-times 
11. Sam Killermann. (2020). Defining a Metrosexual Male. It's Pronounced Metrosexual. Retrieved from https://www.itspronouncedmetrosexual.com/2011/11/defining-a-metrosexual-male/

12. J.A. Shapira. (2017). A History of Men's Fashion and Style. Hespokestyle.com. Retrieved from https://hespokestyle.com/mensfashion-history-timelinel

13. Fashion Revolution. (2020). The impact of COVID-19 on the people who make our clothes.fashionrevolution.org. Retrieved from https://www.fashionrevolution.org/the-impact-of-covid-19-on-the-people-who-make-our-clothes/

14. Rashi Menda. (2020). Style statements: Here are some changes the fashion industry will see in the post-COVID era. Yourstory.com. Retrieved from https://yourstory.com/weekender/fashion-trends-post-covid-rashi-menda-isu 